Original article

\title{
Factors affecting adherence to guidelines for antithrombotic therapy in elderly patients with atrial fibrillation admitted to internal medicine wards
}

\author{
M. Marcucci ${ }^{\mathrm{a}, *}$, A. Iorio $^{\mathrm{a}}$, A. Nobili ${ }^{\mathrm{b}}$, M. Tettamanti $^{\mathrm{b}}$, L. Pasina $^{\mathrm{b}}$, A. Marengoni $^{\mathrm{c}}$, F. Salerno $^{\mathrm{d}}$, \\ S. Corrao ${ }^{\mathrm{e}}$, P.M. Mannucci ${ }^{\mathrm{f}}$ \\ and on behalf of REPOSI Investigators ${ }^{1}$ \\ a Department of Internal Medicine, University of Perugia, Italy \\ ${ }^{\mathrm{b}}$ Mario Negri Institute for Pharmacological Research, Milan, Italy \\ c Geriatric Unit, Ospedali Civili, Department of Medical and Surgery Sciences, University of Brescia, Italy \\ d Internal Medicine, IRCCS Policlinico San Donato, Department of Medical and Surgery, University of Milan, Italy \\ e Biomedical Department of Internal Medicine, University of Palermo, Italy \\ f Scientific Direction, IRCCS Maggiore Hospital Foundation, Milan, Italy
}

\section{A R T I C L E I N F O}

\section{Article history:}

Received 4 June 2010

Received in revised form 14 July 2010

Accepted 17 July 2010

Available online 15 August 2010

\section{Keywords:}

Antithrombotic prophylaxis

Atrial fibrillation

Vitamin $\mathrm{K}$ antagonists

Platelet agents

\begin{abstract}
A B S T R A C T
Introduction: Current guidelines for ischemic stroke prevention in atrial fibrillation or flutter (AFF) recommend Vitamin K antagonists (VKAs) for patients at high-intermediate risk and aspirin for those at intermediate-low risk. The cost-effectiveness of these treatments was demonstrated also in elderly patients. However, there are several reports that emphasize the underuse of pharmacological prophylaxis of cardio-embolism in patients with AFF in different health care settings.

Aims: To evaluate the adherence to current guidelines on cardio-embolic prophylaxis in elderly ( $>65$ years old) patients admitted with an established diagnosis of AFF to the Italian internal medicine wards participating in REPOSI registry, a project on polypathologies/polytherapies stemming from the collaboration between the Italian Society of Internal Medicine and the Mario Negri Institute of Pharmacological Research; to investigate whether or not hospitalization had an impact on guidelines adherence; to test the role of possible modifiers of VKAs prescription.

Methods: We retrospectively analyzed registry data collected from January to December 2008 and assessed the prevalence of patients with AFF at admission and the prevalence of risk factors for cardio-embolism. After stratifying the patients according to their $\mathrm{CHADS}_{2}$ score the percentage of appropriateness of antithrombotic therapy prescription was evaluated both at admission and at discharge. Univariable and multivariable logistic regression models were employed to verify whether or not socio-demographic (age $>80$ years, living alone) and clinical features (previous or recent bleeding, cranio-facial trauma, cancer, dementia) modified the frequency and modalities of antithrombotic drugs prescription at admission and discharge.

Results: Among the 1332 REPOSI patients, 247 were admitted with AFF. At admission, $\mathrm{CHADS}_{2}$ score was $\geq 2$ in $68.4 \%$ of patients, at discharge in $75.9 \%$. Among patients with AFF $26.5 \%$ at admission and $32.8 \%$ at discharge were not on any antithrombotic therapy, and $43.7 \%$ at admission and $40.9 \%$ at discharge were not taking an appropriate therapy according to the $\mathrm{CHADS}_{2}$ score. The higher the level of cardio-embolic risk the higher was the percentage of antiplatelet- but not of VKAs-treated patients. At admission or at discharge, both at univariable and at multivariable logistic regression, only an age $>80$ years and a diagnosis of cancer, previous or active, had a statistically significant negative effect on VKAs prescription. Moreover, only a positive history of bleeding events (past or present) was independently associated to no VKA prescription at discharge in patients who were on VKA therapy at admission. If heparin was considered as an appropriate therapy for patients with indication for VKAs, the percentage of patients admitted or discharged on appropriate therapy became respectively $43.7 \%$ and $53.4 \%$.

Conclusion: Among elderly patients admitted with a diagnosis of AFF to internal medicine wards, an appropriate antithrombotic prophylaxis was taken by less than $50 \%$, with an underuse of VKAs prescription independently of the level of cardio-embolic risk. Hospitalization did not improve the adherence to guidelines.
\end{abstract} (c) 2010 European Federation of Internal Medicine. Published by Elsevier B.V. All rights reserved.

* Corresponding author.

E-mail address: pmmannucci@libero.it (P.M. Mannucci).

1 REPOSI denotes REgistro POliterapie Società Italiana di Medicina Interna. 


\section{Introduction}

Atrial fibrillation or flutter (AFF) is a common and clinically relevant disorder affecting mainly elderly people. Its prevalence rapidly increases after the sixth decade of life and is about $10 \%$ in people more than 80 years of age [1-3]. AFF is associated with a $4-5$ fold increase in the risk of ischemic stroke at all ages, and the percentage of ischemic strokes attributable to AFF rises with advancing age up to nearly $25 \%$ in the class from 80 to 89 years [4-6]. In patients with AFF oral anticoagulant therapy with vitamin $\mathrm{K}$ antagonists (VKAs) prevents cardio-embolism, with a relative risk reduction of ischemic stroke of more than $60 \%[7,8]$. Unfortunately, in the frame of clinical trials VKAs also increase the risk of major bleeding by $0.3-0.5 \% /$ year, and the risk of intracranial hemorrhage by $0.2 \% /$ year [7,9]. Bleeding rates are even higher outside the boundaries of controlled trials [10,11]. Major determinants of the risk of bleeding are intensity of anticoagulation (measured by means of the International Normalized Ratio of the prothrombin time, INR), unpredictable anticoagulant effect in the induction phase (first 3 months of treatment) [12-14] and some patient characteristics (age, history of bleeding, comorbidities such as malignancy and renal failure) [15-18]. Also aspirin is effective in preventing cardio-embolism, but 3-fold less than VKAs and with no clear advantage in terms of safety [8]. It is not yet clear if the combination of aspirin and VKAs is more effective than either drug in patients with AFF, but it does definitely increase the risk of bleeding [19]. Finally, the only other antiplatelet agents evaluated in AFF were indobufen and clopidogrel. Indobufen had no advantage when compared to warfarin versus a composite clinical end-point including incident stroke [20]. In patients not eligible for VKAs, the addition of clopidogrel to aspirin reduced the risk of major vascular events, especially stroke, but increased the rate of major bleeding [21], the combination being clearly less effective than VKAs alone [22]. Extended-release dipyridamole plus aspirin were compared to clopidogrel for recurrent stroke in patients with a recent ischemic stroke, with no evidence of superiority of either treatment, not even in the subgroup of patients with a stroke of cardio-embolic origin (near $2 \%$ in each arm) [23].

With this as background, most guidelines currently recommend antithrombotic prophylaxis for patients with paroxysmal and permanent AFF, tailored according to the level of cardio-embolic risk as calculated on the basis of known risk factors for ischemic stroke in AFF [24-26]. Despite growing use of VKAs in patients with AFF from early 1980s to mid-1990s, and although the cost-effectiveness of this prophylactic strategy is clearly demonstrated particularly in elderly people [27,28], the adherence to guidelines is still very poor in different healthcare settings (community, in- and outpatients, nursing homes, academic hospitals), with a clear trend for decreasing use of VKAs with increasing age [29-35].

The aim of this analysis was to assess the modalities of cardioembolic prophylaxis and to verify the adherence to guidelines in patients with AFF at admission to and at discharge from Italian internal medicine wards participating to a prospective registry of elderly patients. We also attempted to establish which factors modified adherence to antithrombotic therapy.

\section{Methods}

\subsection{Data collection}

We analyzed retrospectively the data collected from January 2008 to December 2008 in the frame of REPOSI, an independent research project on polypathologies/polytherapies based upon a prospective registry and stemming from the collaboration between the Italian Society of Internal Medicine (SIMI) and the Mario Negri Institute for Pharmacological Research. The project has set up a network of internal medicine and geriatric wards that chose to participate to the registry in order to generate information about elderly patients affected by multiple diseases and prescribed with multiple drugs. Patients were eligible for REPOSI if: 1$)$ they were admitted to one of the 36 participating Italian internal medicine $(n=32)$ and geriatric wards $(=4)$ during one of the 4 weeks chosen for recruitment (in February, June, September and December 2008); 2) their age was 65 years or older; 3 ) they gave informed consent. Each ward had to enroll at least the first 10 consecutive eligible patients during each index week. For each patient a web-based case report form was filled in with information about socio-demographic features, cause of hospitalization, diagnoses and treatment both at admission and at discharge, clinical events during hospitalization and outcome. A final database was created, checked and cleaned at the Mario Negri Institute for Pharmacological Research and shared with all the participants in the project. The management of the central repository and the participation of the recruiting units to the registry were voluntary and not sponsored.

\subsection{Study population}

For the purpose of this analysis we selected among the patients recruited in REPOSI only those who had a diagnosis of AFF (paroxysmal or persistent) at admission. The patients were identified in the database by the International Classification of Diseases - Ninth Revision (ICD9) codes 427.31 or 427.32 . Patients with AFF as a new diagnosis during the index hospitalization were not included in this analysis in order to make the study population more homogeneous, to focus on the longterm pharmacological prophylaxis of cardio-embolism and to investigate whether or not hospitalization affected the frequency and modality of application of antithrombotic prophylaxis.

\subsection{Assessment of cardio-embolic risk and appropriateness of antithrombotic prophylaxis}

In order to classify patients with AFF according to their risk of cardio-embolic events, we used the $\mathrm{CHADS}_{2}$ score $[7,36]$ that assigns one point each of the following risk factors: congestive heart failure or a severe impairment of left ventricular systolic function (C); history of hypertension $(\mathrm{H})$; age $>75$ years $(A)$; diabetes mellitus (D). Two points are attributed to a prior ischemic stroke, TIA or systemic embolism $\left(\mathrm{S}_{2}\right)$. The higher is the total score for a patient, the higher is his/her risk of cardio-embolism. The $\mathrm{CHADS}_{2}$ score was calculated at admission to the hospital for the included patients by looking for the risk factors in the database sections collecting demographic characteristics and diagnoses (ICD9 classification). The $\mathrm{CHADS}_{2}$ score was recalculated at discharge and updated if further risk factors were newly detected during hospitalization.

For the evaluation of antithrombotic prophylaxis prescribed at admission and at discharge, we considered as anticoagulants VKAs and as antiplatelet drugs aspirin, clopidogrel, ticlopidine and other agents with antiaggregating action; they were coded in the database section collecting treatment data according to the Anatomical Therapeutic Chemical (ATC) classification as B01AA and B01AC, respectively. Since our goal was to focus on long term cardio-embolic prophylaxis, before analysis of the database we chose not to consider in the analysis any parenteral antithrombotic agents given during hospitalization.

Practice guidelines of the American College of Chest Physicians (ACCP) (8th edition, 2008) [25] were followed to assess the appropriateness of antithrombotic prescription based on the cardioembolic risk. The ACCP recommends long-term anticoagulation with VKAs for patients with AFF and $\mathrm{CHADS}_{2}$ score $\geq 1$ (recommendation grade $1 \mathrm{~A}$ ); for patients with $\mathrm{CHADS}_{2}$ score equal to 1 , aspirin (at a dose of $75-325 \mathrm{mg} /$ day) is proposed as an alternative (grade $1 \mathrm{~B}$ ). Aspirin is also recommended for patients with a low risk of ischemic stroke $\left(\mathrm{CHADS}_{2}=0\right)$. Similar recommendations are contained in the practice guidelines of the American College of Cardiology/American 
Heart Association/European Society of Cardiology (ACC/AHA/ESC) [24].

\subsection{Modifiers of VKAs prescription}

We examined the REPOSI database to search for factors that might be involved in making the clinical decision process or reduce patient compliance, on the basis of literature reports $[9,37,38]$ and clinical judgment, and we tested their association with VKAs intake at admission to the hospital and prescription at discharge. The REPOSI database provided data on the following variables: sex, age $>80$ years, living alone, taking multiple drugs (more than 8 ), bleedings (total and intracranial), cranio-facial traumas related to falls, solid and hematologic tumors, vertigo/vertiginous syndromes/dizziness/ataxia/difficult walking (e.g. a high risk of fall), dementia/cognitive impairment, being bedridden/immobilization syndrome. This information was recorded at hospital admission or as having occurred thereafter during the hospital stay. The diseases were identified in the database according to the ICD9 classification.

\subsection{Statistical analysis}

The prevalence of patients with a preexisting diagnosis of AFF at hospital admission was calculated on all patients included in the REPOSI registry. For patients with AFF we calculated: 1) the prevalence of risk factors for cardio-embolism at admission and at discharge; 2) the prevalence of factors likely to influence VKAs prescription; 3) the prevalence of prescription of antithrombotic regimens in different $\mathrm{CHADS}_{2}$ risk groups, and the crude rate of appropriate prescription of VKAs before and after hospitalization.

Univariable and multivariable logistic regressions (including all the possible modifiers listed above) were modeled to test the association between prescription of VKAs in patients with AFF (outcome variable) and socio-demographic or clinical characteristics. We formally tested for a centre effect on the type of therapy prescribed at discharge by using a set of dummy variables in the logistic model. In order to take into account the multi-center origin of the REPOSI data, we planned to adopt robust variance estimates that were obtained in all regression models by the Huber/White/sandwich estimator which considers observations as independent across groups (centers).

Two sensitivity analyses were performed: 1) the rate of appropriate prescription of antithrombotic therapy in different CHADS $_{2}$ risk groups was recalculated after excluding patients with factors found to be associated with lack of VKA prescription; 2) the rate of appropriate prescription of antithrombotic therapy and the effect of the possible modifiers of VKAs prescription were recalculated after considering heparin as an appropriate therapy for patients with an indication for VKAs.

Patients died during hospitalization were excluded from the analyses at discharge. Patients coming from or moving to other wards or nursing homes were excluded from the analyses on predictors of VKAs prescription, in order to make more homogeneous the sample analyzed.

STATA was used to perform all the analyses (version 9.2, Statacorp, College Station, Tx, US).

\section{Results}

During the 4 weeks selected for the REPOSI data collection, the 36 participating wards enrolled 1332 patients. The majority of these patients came from their homes $(n=1178,88.4 \%)$ after attending or not the emergency room; others were transferred to the internal medicine or geriatric ward from another ward $(n=83,6.0 \%)$ or from a nursing home $(n=48,3.6 \%)$; for 23 patients data about origin were missing. Sixty-six of 1332 patients died during hospitalization (5.0\%), 111 were moved to another ward/hospital, to nursing homes or rehabilitation structures (8.3\%), 1155 were discharged back home (86.7\%). Fig. 1 shows the steps that led to the selection of the population included in this analysis from the whole REPOSI study population. One hundred and twenty-seven were males (51.4\%), 120 were females (48.6\%); they had a mean age of $81.3 \pm 7.5$ years (median 81 years, range $66-100$ ).

\subsection{Cardio-embolic risk stratification and antithrombotic therapy}

There was a high prevalence of cardio-embolic risk factors among patients with AFF. One hundred ninety-five patients (78.9\%) were more than 75 years old. At admission, 144/247 (58.3\%) had hypertension, 63/247 (25.5\%) were diabetic, 65/247 (26.3\%) had a severe impairment of left ventricular systolic function, 11/247 (4.4\%) reported a prior episode of ischemic stroke, TIA or systemic embolism in their clinical history.

Table 1 shows the stratification of the patients with a known diagnosis of AFF according to $\mathrm{CHADS}_{2}$ score, at admission and at discharge, and the prevalence of antithrombotic strategies/no therapy in the different risk groups. Considering inappropriate a prophylactic strategy adopted without following the current guidelines according to the assessed level of risk, and assuming that the other antiplatelet agents were equivalent in efficacy to aspirin, the proportion of patients on appropriate antithrombotic therapy at admission was $107 / 245$ (43.7\%), and 56/168 (33.3\%) when including only patients with $\mathrm{CHADS}_{2}$ score $\geq 2$; at discharge, 95/232 (40.9\%), and 64/176 (36.4\%) when including only patients with $\mathrm{CHADS}_{2}$ score $\geq 2$. Very similar results at admission and discharge were also obtained when patients coming from and moved to another hospital ward were excluded. When data at admission and discharge for patients with higher cardio-embolic risk $\left(\mathrm{CHADS}_{2}\right.$ score $\geq 2$ ) were merged, 15/104 (14.4\%) of patients not taking VKAs at admission were prescribed with this therapy at discharge and 12/57 (21.0\%) of patients taking this therapy at admission were not prescribed it at discharge.

\subsubsection{Association between possible modifiers and VKAs prescription}

Table 2 shows the antithrombotic therapies used in the presence of these possible modifiers (results are limited to most prevalent ones: number of cases at least 30). Fig. 2 (based upon data obtained at admission) and Fig. 3 (at discharge) compare the prevalence of the possible modifiers in patients on therapy and in those not on therapy with VKAs. At admission and discharge, only an age $>80$ years and a diagnosis of cancer (previous or still active), had a statistically significant (or close to statistical significance in patients with $\mathrm{CHADS}_{2}$ score $\geq 2$ ) negative effect on VKAs prescription at univariable logistic regression. Patients already admitted or discharged while taking more than 8 drugs were more likely on therapy with VKAs, with a statistically significant association.

When tested in multivariable regression accounting for the multicentre study design and including all the possible predictors (sex, age $>80$ years, living alone, taking $>8$ drugs, bleedings, cranio-facial traumas, solid and hematologic tumors, risk of fall, dementia), age and malignancy retained their significant negative effect both at admission and discharge. Polypharmacotherapy was not statistically significant associated with prescription of VKAs. In patients with $\mathrm{CHADS}_{2}$ score $\geq 2$, malignancy had a non significant negative association, both at admission and at discharge; age $>80$ years had a statistically significant negative effect at discharge; living alone was associated with statistically significance to prescription of VKAs at discharge.

None of the centers had a significant effect on prescription of VKAs at discharge (likelihood-ratio test for the model accounting for the centre effect 38.6, $p=0.09$ ).

3.1.2. Association between modifiers and in hospital withdrawal of VKAs

When we analyzed the role of possible modifier of VKA prescription at discharge in patients on VKA therapy at admission, only a positive history for bleeding events (past or occurred during hospitalization) 


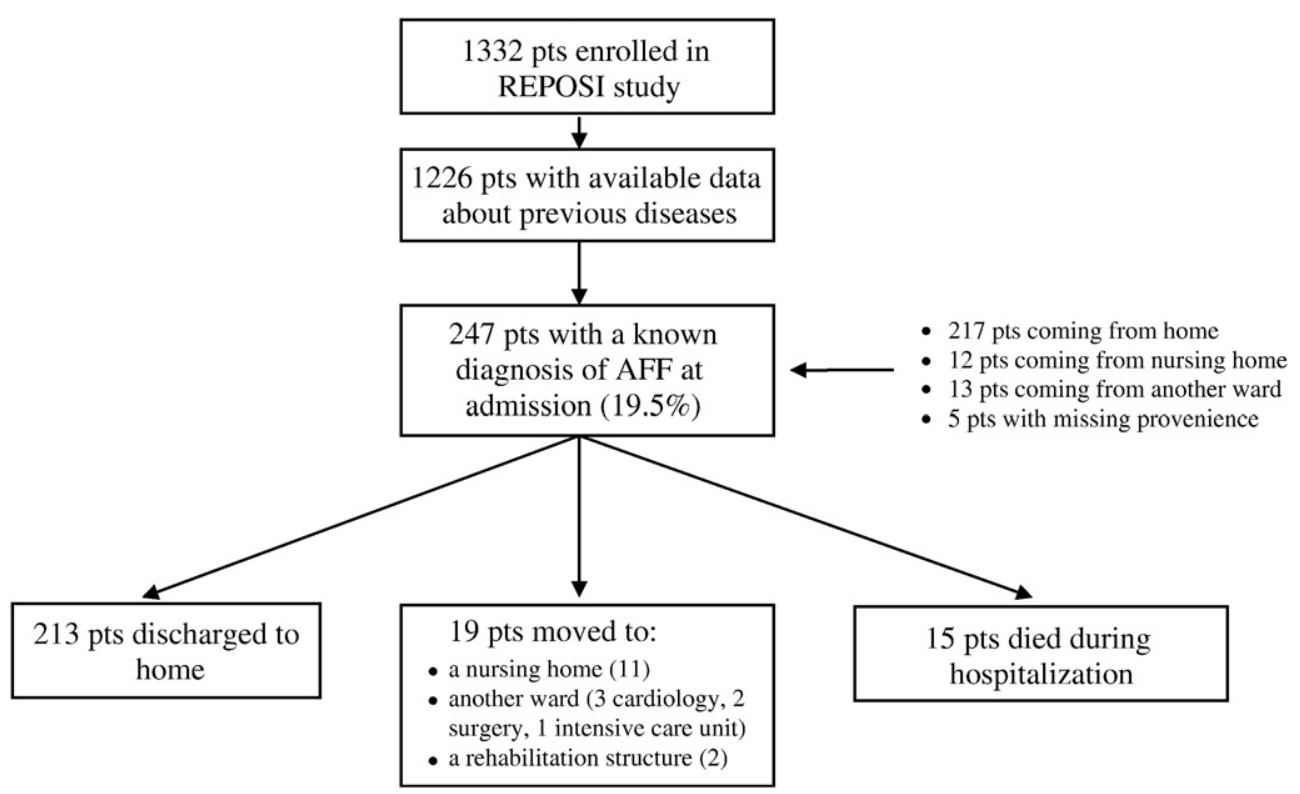

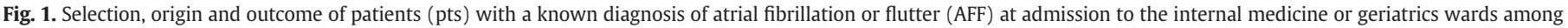
participants in REPOSI.

had a significant negative effect in univariable regression (OR 0.04, $95 \%$ confidence intervals $<0.01-0.38 ; p$ value $=0.005$ ); the OR adjusted for the other covariates was 0.09 (95\% confidence intervals 0.01 $1.01 ; p$ value $=0.051)$.

\subsection{Sensitivity analyses}

We first recalculated rates of appropriate prescription after removal of patients with modifiers that were negatively associated to VKAs prescription. When patients more than 80 years old and with cancer were excluded from the analysis, the frequency of adherence to guidelines was $57.1 \%$ at admission (50.0\% among patients with $\mathrm{CHADS}_{2}$ score $\geq 2$ ) and $56.4 \%$ at discharge (56.1\% among patients with $\mathrm{CHADS}_{2}$ score $\geq 2$ ). We then tested the usage of heparin in patients with indication for VKAs therapy. At admission, 11/147 (7.5\%) patients with $\mathrm{CHADS}_{2}$ score $\geq 1$ not taking VKAs were on therapy with heparin ( 6 out of these coming from their home). Heparin was prescribed to 37/141 (26.2\%) patients with CHADS $_{2}$ score $\geq 1$ discharged without VKAs prescription (29 out of these discharged

Table 1

Percentages of patients with AFF according to their level of cardio-embolic risk and to the type of antithrombotic therapy prescribed at admission to hospital and at discharge (in brackets).

\begin{tabular}{ccllll}
\hline & $\begin{array}{l}\text { All } N=245^{\mathrm{a}} \\
\left(232^{\mathrm{b}}\right)\end{array}$ & VKAs & Aps $^{\mathrm{c}}$ & ${\text { VKAs }+\mathrm{APs}^{\mathrm{d}}}$ & No therapy \\
\hline $\begin{array}{c}\text { All } N=245^{\mathrm{a}} \\
\left(232^{\mathrm{b}}\right)\end{array}$ & & $33.9(34.5)$ & $37.1(30.6)$ & $2.5(2.2)$ & $26.5(32.8)$ \\
$\mathrm{CHADS}_{2} \geq 2$ & $68.4(75.9)$ & $30.9(34.1)$ & $40.5(34.1)$ & $2.4(2.3)$ & $26.2(29.5)$ \\
$\mathrm{CHADS}_{2}=1$ & $25.9(21.1)$ & $42.9(38.8)$ & $31.7(18.4)$ & $1.6(2.0)$ & $23.8(40.8)$ \\
$\mathrm{CHADS}_{2}=0$ & $5.7(3.0)$ & $28.6(14.3)$ & $21.4(28.6)$ & $7.1(0)$ & $42.9(57.1)$ \\
\hline
\end{tabular}

VKAs, Vitamin K Antagonists; APs, Antiplatelet drugs.

a 2 patients with missing data on therapy.

b 15 patients died during hospitalization.

c Among patients on therapy with an AP at admission, 77.3\% were on aspirin, 21.6\% were on ticlopidine; only 1 patient $(0.01 \%)$ was on indobufen. At discharge, $72.5 \%$ were on aspirin, $26.1 \%$ were on ticlopidine; the patient on therapy with indobufen was confirmed with this drug. 5 patients on therapy with two APs: at admission, 2 were on aspirin and clopidogrel, 1 was on aspirin and ticlopidine; 2 were discharged with double antiplatelet therapy (aspirin and clopidogrel).

d At admission, half of patients on therapy with VKAs plus an AP were on aspirin, another half on ticlopidine; all the patients discharged with VKAs plus an antiplatelet were on aspirin. to home). Considering arbitrarily heparin an appropriate therapy for patients with indication for VKAs, the proportion of patients on appropriate therapy became 107/245 (43.7\%) at admission and 132/ 247 (53.4\%) at discharge.

\section{Discussion}

In this retrospective analysis of the REPOSI data, obtained in a prospective cohort of elderly patients with a known diagnosis of AFF hospitalized in Italian internal medicine and geriatric wards in the year 2008, cardio-embolic prophylaxis was far from adherence to the ACCP guidelines in more than half of the cases, both before and after hospitalization. In particular, VKAs were underused irrespective of the level of cardio-embolic risk, and hospitalization did not lead to an improvement in the rate of adherence to guidelines. When investigating the possible role of patient demographic or clinical features on VKAs intake/prescription, a statistically significant inverse association was found between VKA prescription and both an age $>80$ years old and a past or recent history of malignancy, and between a past or recent history of bleeding and VKAs withdrawal at discharge in patients who were taking VKAs at admission.

Our results are in substancial accordance with those obtained in other Italian clinical settings. Ageno et al. [39] reported a very low frequency of antithrombotic therapy among hospitalized patients with AFF one year after discharge. Monte et al. [40] found low rates of VKAs prescription and an association between the underuse of antithrombotic agents after discharge and a poorer outcome. Ferro et al. [41] described a similar scenario among patients at high cardio-embolic risk at admission to a single clinical centre. Our approach provides a more comprehensive evaluation, because data were collected both at admission and discharge with the aim to investigate both the attitude to prescription at the primary care level and any variation related to hospitalization. Even if we were not able to verify if not being on VKAs at admission was due to lack of medical prescription or to poor patient compliance, we found rates of prescription of VKAs/antiplatelet drugs/no therapy very similar to those published by Mazzaglia et al. [42], who analyzed primary care data. Like them, an increasing use of antiplatelet drugs, but not of VKAs, was found in association with a worsening cardio-embolic risk profile. Another novelty of our study consists in having collected data from several hospitals located in different regions of Italy. Finally, the absence of any priori exclusion criteria (e.g. no upper limit of age for 
Table 2

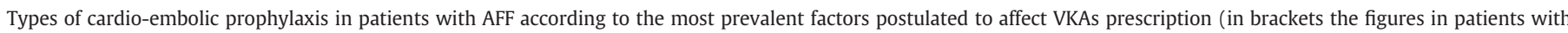
$\left.\mathrm{CHADS}_{2} \geq 2\right)^{*}$

\begin{tabular}{|c|c|c|c|c|c|c|c|c|}
\hline & \multicolumn{4}{|l|}{ At admission } & \multicolumn{4}{|l|}{ At discharge } \\
\hline & \multicolumn{4}{|c|}{$\left(N=217 ; \mathrm{CHADS}_{2} \geq 2: N=146\right)$} & \multicolumn{4}{|c|}{$\left(N=213 ; \mathrm{CHADS}_{2} \geq 2: N=162\right)$} \\
\hline & No therapy & APs & VKAs or VKAs + APs & Total number & No therapy & APs & VKAs or VKAs + APs & Total number \\
\hline Age $>80$ years, $\%$ & $19.5(21.6)$ & $51.3(50.0)$ & $29.2(28.4)$ & $115(89)$ & $28.3(27.4)$ & $42.5(45.3)$ & $29.2(27.4)$ & $113(95)$ \\
\hline Male, \% & $23.5(24.1)$ & $37.4(40.5)$ & $39.1(35.4)$ & $115(79)$ & $29.2(24.1)$ & $32.7(36.8)$ & $38.0(39.1)$ & $113(87)$ \\
\hline Female, \% & $26.0(25.8)$ & $37.0(39.4)$ & $37.0(34.8)$ & $100(66)$ & $34.0(32.0)$ & $28.0(33.3)$ & $38.0(34.7)$ & $100(75)$ \\
\hline Living alone, $\%$ & $38.2(38.1)$ & $23.6(28.6)$ & $38.2(33.3)$ & $56(43)$ & $30.8(26.8)$ & $21.1(26.8)$ & $48.1(46.4)$ & $52(41)$ \\
\hline Taking $>8$ drugs, $\%$ & $16.7(18.5)$ & $27.8(33.3)$ & $55.5(48.1)$ & $36(27)$ & $14.0(14.6)$ & $34.0(41.5)$ & $52.0(43.9)$ & $50(41)$ \\
\hline Malignancy, \% & $48.5(50.0)$ & $33.3(37.5)$ & $18.2(12.5)$ & $33(16)$ & $50.0(40.0)$ & $32.5(40.0)$ & $17.5(20.0)$ & $40(25)$ \\
\hline
\end{tabular}

VKAs, Vitamin K Antagonists; APs, Antiplatelet drugs; AFF, Atrial Fibrillation Flutter.

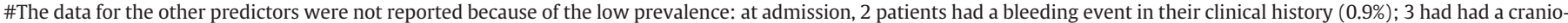

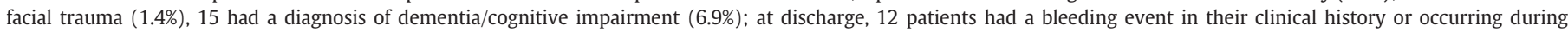

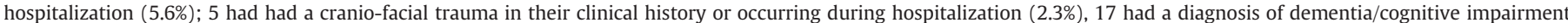

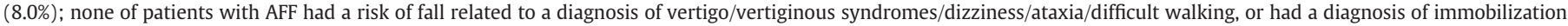
syndrome, either at admission or at discharge.

*Patients coming from or moved to other wards or nursing homes were excluded.

inclusion) and the internal medicine context allowed us to analyze an elderly population of complex patients on multiple drug therapy that better reflects those usually managed in internal medicine wards.

One of the main findings is the lack of a significant impact of hospitalization on the adherence to current guidelines. The proportion of patients with $\mathrm{CHADS}_{2}$ score $\geq 2$ on therapy with VKAs actually increased at discharge as compared to admission (while the proportion of patients with $\mathrm{CHADS}_{2}$ score $\geq 2$ on therapy with antiplatelet decreased), but the absolute percentage was still unsatisfactory and the percentage of patients discharged without any antithrombotic therapy was surprisingly high, irrespective of the level of cardioembolic risk. On the other hand, there were a relevant percentage of patients with $\mathrm{CHADS}_{2}$ score $=0$ who were on therapy with VKAs even if not recommended (and none of these had other diseases justifying anticoagulant prescription). Our results suggest that the level of cardioembolic risk is not the main (or at least not the only), determinant of the therapeutic choice in this population of elderly patients. On the other hand, cardio-embolic risk factors should be considered by the physician as factors associated to the risk of bleeding, because all the bleeding events recorded in this study among AFF patients did occur in patients with $\mathrm{CHADS}_{2}$ score $\geq 1$.

We only partially confirmed the role of some clinical and sociodemographic factors as negative modifiers of VKAs prescription. We found a trend to not prescribe VKAs in patients older than 80 years, even if older age is a known risk factor for cardio-embolic events.

\section{a) All Patients}

\begin{tabular}{|c|c|c|}
\hline modifiers & & $\mathrm{OR}(95 \% \mathrm{Cl})$ \\
\hline sex (male) & & $1.09(0.63,1.90)$ \\
\hline age $>80$ & $\rightarrow$ & $0.45(0.25,0.78)^{*}$ \\
\hline living alone & & $1.01(0.54,1.90)$ \\
\hline taking $>8$ drugs & & $2.36(1.14,4.87)^{\ddagger}$ \\
\hline bleedings & & $1.63(0.10,26.41) \#$ \\
\hline cranio-facial trauma & & $\rightarrow 11.75(0.60,230.54) \dagger$ \\
\hline malignancy & $\longrightarrow$ & $0.31(0.12,0.79) \int$ \\
\hline dementia & & $1.09(0.37,3.18)$ \\
\hline
\end{tabular}

These results are perhaps explained by the physician concern, giving more weight to the high age-related risk of bleeding and to the low compliance in elderly patients than to the similarly high age-related cardio-embolic risk. This finding holds a particular relevance considering the recent debate on the development a new scoring system based upon age alone as an independent predictor of high cardio-embolic risk [43]. Cancer patients with AFF might be less frequently eligible to oral anticoagulation because of a higher risk of bleeding, or because of the fear of drug interaction with chemotherapy or analgesics. Sometime the shorter life expectancy, which magnifies the problems related to the management of VKA therapy, might lead the physician to choose heparin for cardio-embolic prophylaxis in cancer patients even if this choice is not evidence-based. This reasoning could explain the results of our sensitivity analysis on the use of heparin as equivalent to VKAs. The role of these modifiers of VKAs prescription focuses the attention on the issue of the safety. Previous reports have demonstrated that in clinical practice patients with AFF often have characteristics that led to their exclusion from the trials that are the basis of available scientific guidelines [44]. This could be translated as having a different safety profile compared to the patients included in those trials, and could make reasonable not to treat them even in presence of a high cardio-embolic risk.

One of the limitations of our study is the relatively small size of the sample and hence the low power of some analyses, especially when the role of some predictors poorly represented in the database was

\section{b) Patients with $\mathrm{CHADS}_{2}$ score $\geq 2$}

\begin{tabular}{|c|c|}
\hline modifiers & $\mathrm{OR}(95 \% \mathrm{Cl})$ \\
\hline sex (male) & $1.03(0.52,2.04)$ \\
\hline age $>80$ & $0.47(0.24,0.95)^{* *}$ \\
\hline living alone & $0.89(0.42,1.90)$ \\
\hline taking $>8$ drugs & $1.95(0.84,4.56)$ \\
\hline bleedings & $1.86(0.11,30.37) \#$ \\
\hline cranio-facial trauma & $-13.64(0.69,269.42) \dagger$ \\
\hline malignancy & $0.23(0.05,1.07) \iint$ \\
\hline dementia & $0.44(0.09,2.15)$ \\
\hline
\end{tabular}

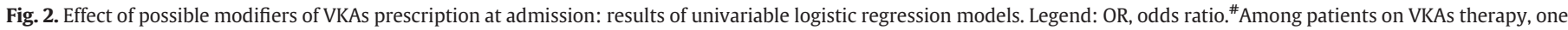

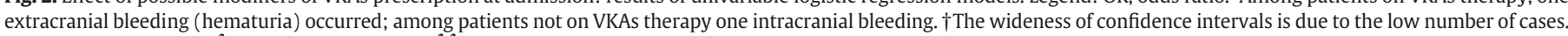
${ }^{*} p=0.008 ; \ddagger p=0.020 ; \int p=0.029 ;{ }^{* *} p=0.063 ; \iint p=0.066$. 


\section{a) All Patients}

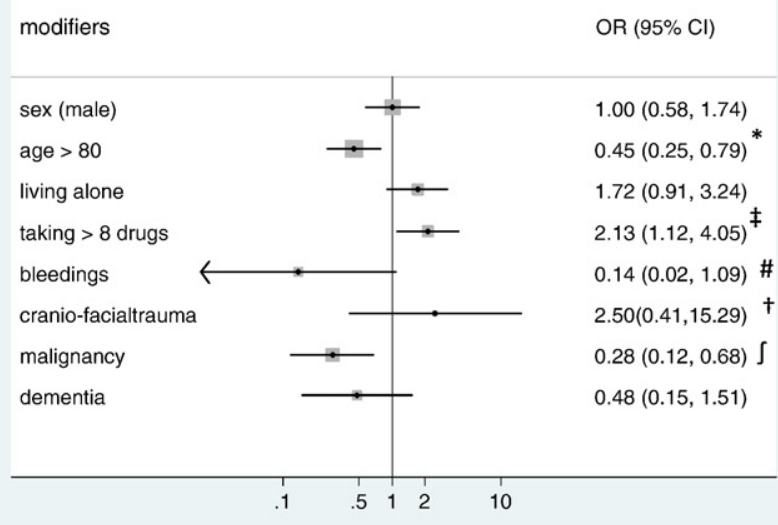

\section{b) Patients with $\mathrm{CHADS}_{2}$ score $\geq 2$}

OR $(95 \% \mathrm{Cl})$

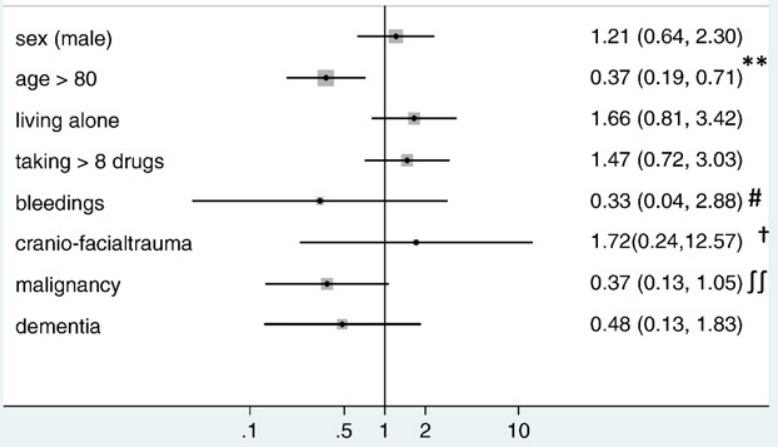

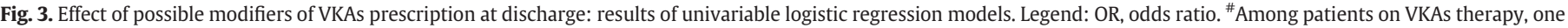

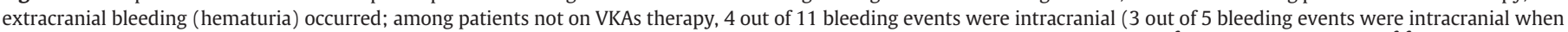
considering patients with $\mathrm{CHADS}_{2} \geq 2$ ). †The width of confidence intervals is due to the low number of cases $p=0.012 ; \ddagger p=0.021 ; \int p=0.004 ; * * p=0.004 ; \iint p=0.053$.

investigated by multivariable regression. This weakness could also have led to apparently meaningless results, such as the association between VKAs prescription and a history of bleeding (see Table 3), even if this finding could be alternatively explained with the high prevalence of minor bleeding inherent in VKAs treatment. Other important limitations derive from the retrospective nature of the analysis, so that only the role of the possible predictors for which data were available in the REPOSI database could be investigated. For example, the choice of ICD9 classification for diagnostic coding did not allow us to distinguish between paroxysmal and permanent AFF, while clinical practice and previous findings [41] suggest that patients with chronic AFF are more likely to be anticoagulated. In general, information provided by a regression analysis, even if adjusted for several covariates, is restricted to the factors put in the equation. In addition, we lacked INR values at admission in order to establish whether or not patients were on effective anticoagulation, and lack of follow up after discharge did not allow us to verify the association between adhesion to guidelines and a more favorable long term outcome, as others have done $[40,45]$. Finally, a further limitation may be the lower than expected prevalence of prior cerebro-vascular events, which was probably due to under-reporting. However, underreporting of cerebro-vascular accidents would have led to underestimation of the $\mathrm{CHADS}_{2}$ score, and hence to bias our results in the direction of greater underestimation on the under-treatment of patients at high cardio-embolic risk.

Despite these limitations, our study adds arguments to the debate on the actual applicability of evidence-based therapeutic guidelines and on the necessity to implement them according to new paradigms [46]. The persistence of a generally poor adherence to guidelines in clinical practice should be a stimulus to increase educational programs for physicians. The situation should also encourage to choose in clinical trials less selected patients, in order to get an evidence based more closely to the real world of the patient with AFF [37,47].

\section{Appendix A. Collaborators and participating units}

Alberto Tedeschi, Raffaella Rossio (Medicina Interna 2, Fondazione IRCCS Ospedale Maggiore, Milano); Guido Moreo, Barbara Ferrari (Medicina Interna 3, Fondazione IRCCS Ospedale Maggiore, Milano); Antonio Mammarella, Valeria Raparelli (Medicina Interna, Università La Sapienza, Roma); Stefania Rondinella, Iolanda Giannico (Medicina Metabolica, Università di Modena e Reggio Emilia); Leonardo Rasciti, Silvia Gualandi (Medicina Interna, Policlinico S. Orsola Malpighi, Bologna); Valter Monzani, Valeria Savojardo (Medicina d'Urgenza,
IRCCS Fondazione Ospedale Maggiore, Milano); Giovanna Fabio, Silvia Colombo (Medicina Interna 1A, Fondazione IRCCS Ospedale Maggiore, Milano); Alessandra Quercioli, Alessandra Barreca (Medicina Interna 1, Università di Genova); Emanuele Durante-Mangoni, Daniela Pinto (Medicina Interna, Seconda Università di Napoli); Elena Incasa, Emanuela Rizzoli (Medicina Interna, Azienda USL, Ferrara); Massimo Vanoli, Gianluca Casella (Medicina Interna, Azienda Ospedaliera di Lecco, Ospedale di Merate); Giuseppe Musca, Olga Cuccurullo (Medicina Interna, P.O. Cetraro, ASP Cosenza); Giuseppe Famularo, Maria Rosaria Sajeva (Medicina Interna 1, Azienda Ospedaliera San Camillo Forlanini, Roma); Antonio Picardi, Dritan Hila (Medicina Clinica-Epatologia, Università Campus Bio-Medico, Roma); Renzo Rozzini, Alessandro Giordano (Fondazione Poliambulanza, Brescia); Antonio Bonelli, Gaetano Dentamaro (Medicina, Ospedale Madonna delle Grazie, Matera); Giulia Gobbo, Massimo Cazzaniga (Medicina Interna, IRCCS Policlinico San Donato, Università di Milano); Piergiorgio Gaudenzi, Lisa Giusto (Medicina ad Alta Rotazione, Azienda Ospedaliera Universitaria, Ferrara); Damiano Rizzoni, Luana Castoldi (Clinica Medica, Università di Brescia); Daniela Mari, Giuliana Micale (Medicina Generale ad indirizzo Geriatrico, IRCCS Istituto Auxologico Italiano, Milano); Emanuele Altomare, Gaetano Serviddio (Medicina Interna, Università di Foggia); Carlo Longhini, Cristian Molino (Clinica Medica, Azienda Mista Ospedaliera Universitaria Sant'Anna, Ferrara); Silvia Deidda, Luciana Maria Cuccuru (Clinica Medica, Azienda Mista Ospedaliera Universitaria, Sassari); Michela Quagliolo, Giuseppe Riccardo Centenaro (Medicina 1, Ospedale di Melegnano, Vizzolo Predabissi, Milano); Anna Laura Pasqui, Luca Puccetti (Medicina Interna, Azienda Ospedaliera Universitaria Le Scotte, Siena); Giampiera Bertolino, Piergiorgio Cavallo (Dipartimento di Medicina Interna, Fondazione IRCCS Policlinico San Matteo, Università degli Studi di Pavia); Daniele Bertolini, Nicola Lucio Liberato (Medicina Interna, Ospedale Carlo Mira, Casorate Primo, Pavia); Antonio Perciccante, Alessia Coralli (Medicina, Ospedale San Giovanni-DecollatoAndisilla, Civita Castellana); Luigi Anastasio, Leonardo Bertucci (Medicina Generale, Ospedale Civile Serra San Bruno); Ana Macura, Davide Matino (Dipartimento di Medicina Interna, Ospedale Santa Maria della Misericordia, Università di Perugia); Cosimo Morabito, Roberto Fava (Medicina, Ospedale Scillesi d'America, Scilla); Antonino Tuttolomondo, Riccardo Di Sciacca (Medicina Interna e Cardioangiologia, Università degli Studi di Palermo); Andrea Semplicini, Giacomo Magagnotti (Clinica Medica 4, Università di Padova); Alessandra Fiorentini, Cristina Tofi (Geriatria, Ospedale di Montefiascone); Carlo Cagnoni, Antonio Manucra (UO Medicina e Primo Soccorso, Ospedale di Bobbio, Azienda USL di Piacenza); Giuseppe Romanelli, (UO Geriatria, Spedali Civili di Brescia); Michele Cortellaro, Maria Rachele Meroni (Medicina 3, Ospedale Luigi 
Sacco, Università di Milano); Dionigi Paolo Rossi, Carlo Vergani (Geriatria, Fondazione IRCCS Ospedale Maggiore e Università di Milano).

\section{Learning points}

- Antithrombotic prophylaxis in patients with AFF is known to be efficacious and safe when prescribed according to the degree of cardio-embolic risk.

- There is evidence in different healthcare settings of a low rate of adherence to guidelines on antithrombotic therapy for patients with AFF.

- We confirmed the underuse of antithrombotic therapy, namely of VKA, among elderly patients with AFF admitted to 36 Italian internal medicine wards, without a positive impact of hospital admission on the rate of adherence to guidelines.

- Considering that various socio-demographic and clinical factors other than those used to stratify the cardio-embolic risk may influence the decision to prescribe VKAs, a significant association was found between a history of cancer or previous and current bleeding and omission of VKAs prescription at admission to the hospital as well as at discharge.

\section{References}

[1] Feinberg WM, Blackshear JL, Laupacis A, Kronmal R, Hart RG. Prevalence, age distribution, and gender of patients with atrial fibrillation analysis and implications. Arch Intern Med 1995;155:469-73.

[2] Miyasaka Y, Barnes ME, Gersh BJ, Cha SS, Bailey KR, Abhayaratna WP, et al. Secular trends in incidence of atrial fibrillation in Olmsted County, Minnesota, 1980 to 2000 , and implications on the projections for future prevalence. Circulation 2006;114:119-25.

[3] Bilato C, Corti MC, Baggio G, Rampazzo D, Cutolo A, Iliceto S, et al. Prevalence, functional impact, and mortality of atrial fibrillation in an older Italian population (from the Pro.V.A. study). Am J Cardiol 2009;104:1092-7.

[4] Wolf PA, Abbott RD, Kannel WB. Atrial fibrillation: a major contributor to stroke in the elderly. The Framingham Study Arch Intern Med 1987;147:1561-4.

[5] Yuan Z, Bowlin S, Einstadter D, Cebul RD, Conners Jr AR, Rimm AA. Atrial fibrillation as a risk factor for stroke: a retrospective cohort study of hospitalized Medicare beneficiaries. Am J Public Health 1998;88:395-400.

[6] Lakshminarayan K, Solid CA, Collins AJ, Anderson DC, Herzog CA. Atrial fibrillation and stroke in the general medicare population: a 10-year perspective (1992 to 2002). Stroke 2006;37:1969-74.

[7] Risk factors for stroke and efficacy of antithrombotic therapy in atrial fibrillation. Analysis of pooled data from five randomized controlled trials. Arch Intern Med 1994:154:1449-57.

[8] Hart RG, Benavente O, McBride R, Pearce LA. Antithrombotic therapy to prevent stroke in patients with atrial fibrillation: a meta-analysis. Ann Intern Med 1999;131: 492-501.

[9] Schulman S, Beyth RJ, Kearon C, Levine MN. Hemorrhagic complications of anticoagulant and thrombolytic treatment: American College of Chest Physicians Evidence-Based Clinical Practice Guidelines (8th Edition). Chest 2008;133: 257S-98S.

[10] Jackson SL, Peterson GM, Vial JH, Daud R, Ang SY. Outcomes in the management of atrial fibrillation: clinical trial results can apply in practice. Intern Med J 2001;31: 329-36.

[11] Abdelhafiz AH, Wheeldon NM. Results of an open-label, prospective study of anticoagulant therapy for atrial fibrillation in an outpatient anticoagulation clinic. Clin Ther 2004;26:1470-8.

[12] Casais P, Luceros AS, Meschengieser S, Fondevila C, Santarelli MT, Lazzari MA. Bleeding risk factors in chronic oral anticoagulation with acenocoumarol. Am J Hematol 2000;63:192-6.

[13] Yamaguchi T. Optimal intensity of warfarin therapy for secondary prevention of stroke in patients with nonvalvular atrial fibrillation : a multicenter, prospective, randomized trial. Japanese Nonvalvular Atrial Fibrillation-Embolism Secondary Prevention Cooperative Study Group. Stroke 2000;31:817-21.

[14] Fang MC, Chang Y, Hylek EM, Rosand J, Greenberg SM, Go AS, et al. Advanced age, anticoagulation intensity, and risk for intracranial hemorrhage among patients taking warfarin for atrial fibrillation. Ann Intern Med 2004;141:745-52.

[15] Palareti G, Leali N, Coccheri S, Poggi M, Manotti C, D'Angelo A, et al. Bleeding complications of oral anticoagulant treatment: an inception-cohort, prospective collaborative study (ISCOAT). Italian Study on Complications of Oral Anticoagulant Therapy Lancet 1996;348:423-8.

[16] White RH, McKittrick T, Takakuwa J, Callahan C, McDonell M, Fihn S. Management and prognosis of life-threatening bleeding during warfarin therapy. National Consortium of Anticoagulation Clinics. Arch Intern Med 1996;156:1197-201.

[17] Hutten BA, Lensing AW, Kraaijenhagen RA, Prins MH. Safety of treatment with oral anticoagulants in the elderly. A systematic review. Drugs Aging 1999;14:303-12.
[18] Pengo V, Legnani C, Noventa F, Palareti G. Oral anticoagulant therapy in patients with nonrheumatic atrial fibrillation and risk of bleeding. A Multicenter Inception Cohort Study. Thromb Haemost 2001;85:418-22.

[19] Donnan GA, Davis SM. Combined aspirin plus warfarin: recent evidence and residual questions. Stroke 2009;40:1946

[20] Morocutti C, Amabile G, Fattapposta F, Nicolosi A, Matteoli S, Trappolini M, et al. Indobufen versus warfarin in the secondary prevention of major vascular events in nonrheumatic atrial fibrillation. SIFA (Studio Italiano Fibrillazione Atriale) Investigators. Stroke 1997;28:1015-21.

[21] Connolly SJ, Pogue J, Hart RG, Hohnloser SH, Pfeffer M, Chrolavicius S, et al. Effect of clopidogrel added to aspirin in patients with atrial fibrillation. $\mathrm{N}$ Engl J Med 2009;360:2066-78.

[22] Connolly S, Pogue J, Hart R, Pfeffer M, Hohnloser S, Chrolavicius S, et al. Clopidogre plus aspirin versus oral anticoagulation for atrial fibrillation in the Atria fibrillation Clopidogrel Trial with Irbesartan for prevention of Vascular Events (ACTIVE W): a randomised controlled trial. Lancet 2006;367:1903-12.

[23] Sacco RL, Diener HC, Yusuf S, Cotton D, Ounpuu S, Lawton WA, et al. Aspirin and extended-release dipyridamole versus clopidogrel for recurrent stroke. N Engl J Med 2008;359:1238-51.

[24] Fuster V, Ryden LE, Cannom DS, Crijns HJ, Curtis AB, Ellenbogen KA, et al. ACC/AHA/ ESC 2006 guidelines for the management of patients with atrial fibrillationexecutive summary: a report of the American College of Cardiology/American Heart Association Task Force on Practice Guidelines and the European Society of Cardiology Committee for Practice Guidelines (Writing Committee to Revise the 2001 Guidelines for the Management of Patients With Atrial Fibrillation). J Am Coll Cardiol 2006;48:854-906.

[25] Singer DE, Albers GW, Dalen JE, Fang MC, Go AS, Halperin JL, et al. Antithrombotic therapy in atrial fibrillation: American College of Chest Physicians Evidence-Based Clinical Practice Guidelines (8th Edition). Chest 2008;133:546S-92S.

[26] Lloyd-Jones D, Adams RJ, Brown TM, Carnethon M, Dai S, De SG, et al. Executive summary: heart disease and stroke statistics-2010 update: a report from the American Heart Association. Circulation 2010;121:948-54.

[27] Caro JJ, Flegel KM, Orejuela ME, Kelley HE, Speckman JL, Migliaccio-Walle K. Anticoagulant prophylaxis against stroke in atrial fibrillation: effectiveness in actual practice. CMAJ 1999;161:493-7.

[28] Singer DE, Chang Y, Fang MC, Borowsky LH, Pomernacki NK, Udaltsova N, et al. The net clinical benefit of warfarin anticoagulation in atrial fibrillation. Ann Intern Med 2009; 151:297-305.

[29] Albers GW, Yim JM, Belew KM, Bittar N, Hattemer CR, Phillips BG, et al. Status of antithrombotic therapy for patients with atrial fibrillation in university hospitals. Arch Intern Med 1996;156:2311-6.

[30] Thromboembolic prophylaxis in 3575 hospitalized patients with atrial fibrillation. The Clinical Quality Improvement Network (CQIN) Investigators. Can J Cardiol 1998;14:695-702.

[31] Go AS, Hylek EM, Borowsky LH, Phillips KA, Selby JV, Singer DE. Warfarin use among ambulatory patients with nonvalvular atrial fibrillation: the anticoagulation and risk factors in atrial fibrillation (ATRIA) study. Ann Intern Med 1999;131: 927-34.

[32] Smith NL, Psaty BM, Furberg CD, White R, Lima JA, Newman AB, et al. Temporal trends in the use of anticoagulants among older adults with atrial fibrillation. Arch Intern Med 1999;159:1574-8

[33] Gage BF, Boechler M, Doggette AL, Fortune G, Flaker GC, Rich MW, et al. Adverse outcomes and predictors of underuse of antithrombotic therapy in medicare beneficiaries with chronic atrial fibrillation. Stroke 2000;31:822-7.

[34] McCormick D, Gurwitz JH, Goldberg RJ, Becker R, Tate JP, Elwell A, et al. Prevalence and quality of warfarin use for patients with atrial fibrillation in the long-term care setting. Arch Intern Med 2001;161:2458-63.

[35] Wittkowsky AK. Effective anticoagulation therapy: defining the gap between clinical studies and clinical practice. Am J Manag Care 2004;10:S297-306

[36] Gage BF, Waterman AD, Shannon W, Boechler M, Rich MW, Radford MJ. Validation of clinical classification schemes for predicting stroke: results from the National Registry of Atrial Fibrillation. JAMA 2001;285:2864-70.

[37] Hylek EM, D'Antonio J, Evans-Molina C, Shea C, Henault LE, Regan S. Translating the results of randomized trials into clinical practice: the challenge of warfarin candidacy among hospitalized elderly patients with atrial fibrillation. Stroke 2006;37:1075-80.

[38] Garwood CL, Corbett TL. Use of anticoagulation in elderly patients with atria fibrillation who are at risk for falls. Ann Pharmacother 2008;42:523-32.

[39] Ageno W, Ambrosini F, Nardo B, Imperiale D, Dentali F, Mera V, et al. Atrial fibrillation and antithrombotic treatment in Italian hospitalized patients: a prospective, observational study. J Thromb Thrombolysis 2001;12:225-30.

[40] Monte S, Macchia A, Pellegrini F, Romero M, Lepore V, D'Ettorre A, et al Antithrombotic treatment is strongly underused despite reducing overall mortality among high-risk elderly patients hospitalized with atrial fibrillation. Eur Heart J 2006;27:2217-23.

[41] Ferro D, Loffredo L, Polimeni L, Violi F. Underuse of oral anticoagulants in patients with nonvalvular atrial fibrillation in Italy. Intern Emerg Med 2007;2:24-8.

[42] Mazzaglia G, Filippi A, Alacqua M, Cowell W, Shakespeare A, Mantovani LG, et al. A national survey of the management of atrial fibrillation with antithrombotic drugs in Italian primary care. Thromb Haemost 2010;103.

[43] Lip GY, Nieuwlaat R, Pisters R, Lane DA, Crijns HJ. Refining clinical risk stratification for predicting stroke and thromboembolism in atrial fibrillation using a novel risk factor-based approach: the euro heart survey on atrial fibrillation. Chest 2010;137: 263-72.

[44] Levi M, Hovingh GK, Cannegieter SC, Vermeulen M, Buller HR, Rosendaal FR. Bleeding in patients receiving vitamin $\mathrm{K}$ antagonists who would have been 
excluded from trials on which the indication for anticoagulation was based. Blood 2008; $111: 4471-6$

[45] Nieuwlaat R, Olsson SB, Lip GY, Camm AJ, Breithardt G, Capucci A, et al. Guidelineadherent antithrombotic treatment is associated with improved outcomes compared with undertreatment in high-risk patients with atrial fibrillation. The Euro Heart Survey on Atrial Fibrillation. Am Heart J 2007;153:1006-12.
[46] Pisters R, de Vos CB, Nieuwlaat R, Crijns HJ. Use and underuse of oral anticoagulation for stroke prevention in atrial fibrillation: old and new paradigms. Semin Thromb Hemost 2009;35:554-9.

[47] Weisbord SD, Whittle J, Brooks RC. Is warfarin really underused in patients with atrial fibrillation? J Gen Intern Med 2001;16:743-9. 